\title{
Capabilities of Combined Positron Emission and Computed Tomography in Neuroendocrine Tumor Diagnostics: First Experience of Russian Synthesis Module 68Ga-DOTA-TATE
}

\author{
DOI: $10.17691 / \mathrm{stm} 2016.8 .4 .07$
}

Received June 30, 2016

M.S. Tlostanova, MD, PhD, Head of Positron Emission Tomography Unit;

M.M. Khodzhibekova, MD, PhD, Radiologist;

A.A. Panfilenko, MD, PhD, Radiologist, Positron Emission Tomography Unit;

A.A. Balabanova, MD, PhD, Radiologist, Positron Emission Tomography Unit;

N.A. Kostenikov, MD, DSc, Head of the Laboratory of Preclinical Studies of Radiopharmaceuticals;

S.V. Shatik, MD, PhD, Head of the Department of Cyclotron Radiopharmaceuticals, Head of the Laboratory

of Radiopharmaceutical Technologies;

V.V. Zaitsev, Leading Process Engineer, Head of Radiopharmaceutical Synthesis Group, Department

of Cyclotron Radiopharmaceuticals;

D.S. Sysoev, Leading Engineer, Department of Cyclotron Radiopharmaceuticals, Head of the Group of Development and Production of Equipment for Nuclear Medicine;

A.A. Stanzhevsky, MD, DSc, Deputy Director for Science;

A.M. Granov, MD, DSc, Professor, Academician of the Russian Academy of Sciences, Director

Russian Research Center for Radiology and Surgical Technologies, Ministry of Health of the Russian Federation, 70 Leningradskaya St., Saint Petersburg, Pesochniy pos., 197758, Russian Federation

The aim of the investigation was to estimate the sensitivity of combined positron emission and computed tomography (PET/CT) in the diagnostics, extension and treatment efficiency of neuroendocrine tumors (NET) using ${ }^{68} \mathrm{Ga}-\mathrm{DOTA}$-TATE as an imaging agent.

Materials and Methods. PET/CT with ${ }^{68} \mathrm{Ga}$-DOTA-TATE was performed in 86 patients with pulmonary and gastrointestinal NET (55 patients underwent it preoperatively, 31 patients - postoperatively). All patients before PET/CT were administered a diagnostic dose of radiopharmaceutical (RP) at the rate of $1.5 \mathrm{MBq}$ per a kilogram of body mass but not less than $100 \mathrm{MBq}$. The developed RP synthesis module (Gerat13-68 $\mathrm{Ga}$ ) enabled to prepare a ready for intravenous administration ${ }^{68} \mathrm{Ga}-\mathrm{DOTA}-\mathrm{TATE}$ within 15 min. Moreover, RP was defined to have a stably high radiochemical yield (over $50 \%$ without decay correction) and the appropriate quality. The patients were scanned 50-60 min after RP injection, the procedure being carried out according to a whole body protocol using PET/CT Discovery 690 (General Electric, USA). The data processing included the image analysis of computed and positron emission tomograms, as well as combined images. The main feature of sst-positive tissue tumor was pathological focal ${ }^{68} \mathrm{Ga}$-DOTA-TATE hyperfixation even if there were no evident structural changes of the organ according to CT findings. In cases when CT showed a tumor but the tumor didn't accumulated RP, its nature was considered to be malignant and the tumor was estimated as sst-negative.

Results. In patients examined before treatment, the sensitivity of PET/CT with ${ }^{68} \mathrm{Ga}-\mathrm{DOTA}$-TATE to diagnose a primary tumor was $90.9 \%$ (50/55). In 3 patients PET showed a sst-negative tumor, in 2 patients a primary mass was not detected after tomography either. In addition, an initially determined stage of the disease was confirmed by PET/CT in 36 of 47 cases (76.6\%). The rest patients were found to have second metastases in different organs and systems.

PET/CT findings in 13 of 31 patients examined after the treatment didn't coincide with those obtained by other techniques. In 6 patients the presence of pathological PR accumulation indicated no positive effect of the therapy, 3 patients were found to have secondary changes, 3 patients were detected to have a significant increase of liver metastases (only in CT), and 1 female patient after surgery appeared to have the disease recurrence in the stump of the left main bronchus.

For contacts: Marina S. Tlostanova, e-mail: tlostanovamarina@gmail.com 
Conclusion. PET/CT with ${ }^{68} \mathrm{Ga}-\mathrm{DOTA}-\mathrm{TATE}$ demonstrated high efficiency in primary tumor detection, the assessment of therapy results and NET extension.

Key words: PET/CT; ${ }^{68} \mathrm{Ga}-\mathrm{DOTA}-\mathrm{TATE} ;$ neuroendocrine tumors; peptide synthesis module Gerat13-68 Ga.

Neuroendocrine tumors (NET) refer to the neoplasms of neuroendocrine cellular system, its main features are synthesis, as well as the deposition of biogenic amine precursors and their subsequent decarboxylation. Taking into account the variety of the tumors, the "neuroendocrine neoplasia" concept has been reconsidered several times, and currently the most common synonyms of the term are "gastroenteropancreatic tumors", "islet cell tumors", "carcinoids", etc.

The findings of statistical studies presented in SEER (Surveillance, Epidemiology, and End Results, National Cancer Institute, USA), as well as in Norwegian Cancer Register (NCR), the largest European database on $\mathrm{NET}$, indicate that in recent years there is a significant growth of NET incidence [1,2]. The main causes are still unclear, however, the mentioned authors link an increasing NET detection rate to the enhancement of diagnostic techniques and novel approaches to the pathology classification. Unfortunately, in the Russian Federation there is no NET register. This fact limits the development of a single algorithm for NET diagnostics and management in our country.

Since NET develop from chromaffin cells of the entodermal canal, according to the embryogenesis classification by E.D. Williams and M. Sanders, it is common practice to systemize them by an origin site: a part of the entodermal canal. So, carcinoids of the lungs, thymus, stomach, proximal duodenal and pancreatic parts refer to the foregut, and are characterized by a high potential of metastatic lesions of skeletal bones, low serotonin production, low histamine secretion, as well as no clear presentation of carcinoid syndrome. The most common conditions in this NET group are insulinomas resulting from the islet apparatus of the pancreas, and in 80-90\% cases are benign tumors.

NET of a distal duodenum, and a proximal large intestine including the appendix referring to the midgut are characterized by an increased production of serotonin and other vasoactive substances (kinines, prostaglandins) causing high incidence rate of carcinoid syndrome, its typical clinical presentation being flushing (blush, tachycardia, fever sensation) and diarrhea.

NET of the sigmoid colon and rectum originate from the hindgut. In these tumors carcinoid syndrome does not develop, however, the incidence of liver metastases is high.

One of the chief tasks in NET detection regardless its location is the determination of a biological potential of tumor aggressiveness. Moreover, the estimation of standard tumor grade criteria (atypia and infiltrative growth) in carcinoids is insufficient. Due to the fact, a number of other parameters are considered. In accordance with the consensus reached by leading European anatomic pathologists at ENETS in 2010, depending on a tumor grade, gastrointestinal (GI) NET are subdivided into three groups (Grade 1-3). G1, G2 tumors include high-differentiated GI NET with intensive expression of chromogranin A and synaptophysin. G3 tumors include low-differentiated neuroendocrine carcinomas with numerous and distant metastases, of large size, with necroses, vascular and nerve invasion, usually mild chromogranin A expression, intensive synaptophysin expression, high myotic index and Ki-67, marked nuclear polymorphism and other signs described in detail by World Health Organization (WHO) in GI NET classification (2000).

According to a current WHO classification (2004), pulmonary NET are subdivided into 4 histological subtypes: high-differentiated typical carcinoids (G1), moderately differentiated atypical carcinoids (G2a), lowdifferentiated (anaplastic) atypical carcinoids (G2b), small cell carcinomas (G3) and large cell neuroendocrine carcinomas (G4).

All NET depending on their ability to produce certain biologically active substances are generally divided into functionally active and inactive. Functionally active NET in various amounts can produce serotonin, chromogranin A, neuron-specific enolase, 5-hydroxytryptofan, synaptophysin, insulin, pancreatic polypeptide, growth hormone, calcitonin, various tachykinins, releasing hormone, platelet-derived growth factor, growth hormone, bombesin, etc. [3]. It is significant that the secretion of a particular hormone is not a stable parameter of a tumor, since many NET can secrete several biologically active substances. Moreover, in the course of disease progression the range of the substances produced can change. Nonfunctioning compounds compared to functionally active have a more aggressive course, since they are found at advanced stages. In these patients for a long period of time there is the increase of biochemical markers only, and there are not any clinical signs, which ultimately result in severe complications [4-7].

To detect a primary tumor and determine the grade of a malignancy, NET patients usually undergo various endoscopic studies including endoscopic ultrasound scanning, computed and magnetic resonance tomography. In recent years a diagnostic algorithm of NET patients frequently includes radionuclide technologies, scintigraphy and/or positron emission tomography combined with computed tomography (PET/CT).

It is generally admitted that PET/CT with a widely used radiopharmaceutical (RP) ${ }^{18} \mathrm{~F}$-fluorodeoxyglucose 
$\left({ }^{18} \mathrm{~F}-\mathrm{FDG}\right)$ enables to detect to high precision most malignancies, determine the extent of a neoplastic process within "the whole body", as well as estimate an early response of a tumor to the treatment provided. At the same time, imaging capabilities of PET/CT with ${ }^{18}$ F-FDG to diagnose high-differentiated NET are limited due to a great number of false-negative data [8-11]. This is due to the fact that a glycolysis level in malignant cells of carcinoids is in direct relationship to tumor biological aggressiveness.

A successful solution of the problems has been facilitated by the onrush of nuclear technologies causing the emergence of radically new receptor specific RP for $\mathrm{PET} / \mathrm{CT}$ providing higher diagnostic accuracy in NET detection than that using ${ }^{18} \mathrm{~F}-\mathrm{FDG}$. The updating process of RP production enabled to develop new radiotracers not by a traditional cyclotron way but using ${ }^{68} \mathrm{Ge} /{ }^{68} \mathrm{Ga}-$ generator. It is significant that the works on developing a medical ${ }^{68} \mathrm{Ge} /{ }^{68} \mathrm{Ga}$-generator in Russia have been carried out in the Institute of biophysics of the Ministry of Health, USSR, since the mid 60ies of the last century. Later, in the 80 ies, there were carried out the studies on germanium and gallium sorption on a large amount of sorbent agents, which were available at that time in our country. As a result, ${ }^{68} \mathrm{Ge} /{ }^{68} \mathrm{Ga}$-generator was designed, which enabled to elute a radionuclide ${ }^{68} \mathrm{Ga}$ in ionic state in the form of chloride complexes. Further, the generator was refined jointly with CJSC "Cyclotron" (Obninsk, Russia) and defended by a RF patent [12], and in 2000 a generator became commercially available.

By now there have been studied a great number of all possible ${ }^{68} \mathrm{Ga}$-labeled bioconjugates, which enable to image physiological processes using PET. In addition, a considerable part of current researches is devoted to the study of the efficacy of labeled ${ }^{68} \mathrm{Ga}$ synthetic analogues of a natural cyclic peptide hormone - somatostatin. It is significant that the main reasons of a substitution of a natural somotostatin for synthetic analogues were its fast cleavage in human blood, as well as an ultra-short half-life, which is a few minutes only. It is common knowledge that in the norm human somatostatin is synthesized by delta cells of the hypothalamus and Langerhans islets of the pancreas in the form of a high molecular weight precursor protein - preprosomatostatin consisting of 116 amino acid residues. The precursor as through posttranslational processing turns into prosomatostatin composed of 92 amino acid residues. Then prosomatostatin changes into somatostatin by means of specific proteolysis.

Currently, there are 6 subtypes of somatostatin tumor receptors (sst1, sst2A, sst2B, sst3, sst4, sst5), which, except sst2, are encoded by different genes [13]. Overexpression of these receptors on the surface of atypical cells is the characteristic of NET that differ them from malignancies of other genesis. It is the characteristic of cells that was taken as a basis of carcinoid imaging, and subsequently NET therapy using radionuclides.

Generally, biomolecules specific to a receptor cellular apparatus and labeled by ${ }^{68} \mathrm{Ga}$ or other radionuclides (metals) unable to form stable bonds between themselves. Therefore, to enhance the stability of biological conjugates it is necessary to introduce bifunctional chelating agents serving as an additional grouping. Currently, chelator DOTA-(1,4,7,10-tetraazacyclododecane-1,4,7,10-tetraacetic acid) is widely used to provide the stability of a resultant biocomplex.

In current medical practice several DOTA-conjugated RP $\quad{ }^{68} \mathrm{Ga}$-DOTA-TATE, ${ }^{68} \mathrm{Ga}-D O T A-N O C, \quad{ }^{68} \mathrm{Ga}$-DOTATOC) are used. The composition of these RP can be described as follows: ${ }^{68} \mathrm{Ga}$ is an isotope (T1/2=67.6 $\left.\mathrm{min}\right)$ obtained by a generator ${ }^{68} \mathrm{Ge} /{ }^{68} \mathrm{Ga}$; DOTA is a chelator; TOC, NOC, TATE are active parts of a biological compound directly bonding to sst-receptors of a typical cell. According to literature data, the mentioned RP depending on an active part of a compound exhibit different affinity to different types of sst-receptors. In addition, the affinity degree of conjugates to certain types of receptors is still a discussible issue [14-16].

The aim of the investigation was to estimate the sensitivity of combined positron emission and computed tomography in the diagnostics, extension and treatment efficiency of neuroendocrine tumors using ${ }^{68} \mathrm{Ga}$-DOTATATE as an imaging agent.

Materials and Methods. From September 2015 to June 2016, on the basis of Russian Research Center for Radiology and Surgical Technologies, Ministry of Health, Russia, 86 patients with NET underwent an integrated radiation examination including PET/CT with ${ }^{68} \mathrm{Ga}$-DOTATATE (See the Table). The study complies with the declaration of Helsinki (adopted in June, 1964 (Helsinki, Finland) and revised in October, 2000 (Edinburg, Scotland)) and was approved by the Ethics Committee of Russian Research Center for Radiology and Surgical Technologies, Ministry of Health, Russia. All patients gave their informed consent.

In our sampling of patients there was no gender predominance. In the overwhelming majority of cases, according to other research techniques, primary tumor site was determined before PET/CT with ${ }^{68} \mathrm{Ga}$-DOTATATE. Only $8.1 \%(7 / 86)$ patients underwent it to detect a primary tumor. According to the morphological analysis, in most patients there were diagnosed locally advanced and disseminated GI NET (mostly, the ileum and pancreas) characterized by primarily moderate and low differentiation of atypical cells (G2, G3). According to laboratory findings, and the results of patients' complaints, in $73.3 \%(63 / 86)$ cases NET were estimated as functionally inactive. The rest of the patients had hypersecretion of various biologically active substances and/or carcinoid syndrome manifestations.

All patients before PET/CT were given a diagnostic dose of ${ }^{68} \mathrm{Ga}$-DOTA-TATE at the rate of $1.5 \mathrm{MBq}$ per a kilogram of body mass, but not less than $100 \mathrm{MBq}$ administered intravenously by a stream fusion. It should be noted, that ${ }^{68} \mathrm{Ga}-\mathrm{DOTA}$-TATE was produced on Gerat13- ${ }^{68} \mathrm{Ga}$ (Figure 1), a Russian ${ }^{68} \mathrm{Ga}$-labeled peptide synthesis module developed by Russian Research Center 
Initial demographic and clinical characteristics of patients with neuroendocrine tumors

\begin{tabular}{|c|c|c|}
\hline \multirow{2}{*}{ Characteristics } & \multicolumn{2}{|c|}{ Number of patients $(n=86)$} \\
\hline & abs. number & percentage \\
\hline $\begin{array}{l}\text { Gender: } \\
\text { males } \\
\text { females }\end{array}$ & $\begin{array}{l}45 \\
41\end{array}$ & $\begin{array}{l}52.3 \\
47.7\end{array}$ \\
\hline $\begin{array}{l}\text { Age (years): } \\
\text { median } \\
\text { range }\end{array}$ & $\begin{array}{c}57 \\
21-69\end{array}$ & - \\
\hline $\begin{array}{l}\text { Tumor grade: } \\
\text { high-differentiated } \\
\text { G1 (Ki-67 } \leq 2 \% \text { ) } \\
\text { G2 (Ki-67 from } 3 \text { to 20\%) } \\
\text { low-differentiated } \\
\text { G3 (Ki-67 is } 21 \% \text { and more) } \\
\text { Gx (Ki-67 is unknown) }\end{array}$ & $\begin{array}{c}8 \\
34 \\
37 \\
7\end{array}$ & $\begin{array}{c}9.3 \\
39.6 \\
43.0 \\
8.1\end{array}$ \\
\hline $\begin{array}{l}\text { Primary site location: } \\
\text { main, lobar bronchi, lungs } \\
\text { small intestine } \\
\text { stomach } \\
\text { pancreas } \\
\text { a primary site is not established }\end{array}$ & $\begin{array}{c}9 \\
23 \\
11 \\
36 \\
7\end{array}$ & $\begin{array}{l}10.5 \\
26.7 \\
12.8 \\
41.9 \\
8.1\end{array}$ \\
\hline $\begin{array}{l}\text { Tumor functionality (there are or there are no clinical } \\
\text { and/or biochemical manifestations of carcinoid syndrome): } \\
\text { functionally active tumor } \\
\text { functionally inactive tumor }\end{array}$ & $\begin{array}{l}23 \\
63\end{array}$ & $\begin{array}{l}26.7 \\
73.3\end{array}$ \\
\hline $\begin{array}{l}\text { Dissemination degree: } \\
\text { localized } \\
\text { locally advanced } \\
\text { disseminated }\end{array}$ & $\begin{array}{l}13 \\
51 \\
22\end{array}$ & $\begin{array}{l}15.1 \\
59.3 \\
25.6\end{array}$ \\
\hline $\begin{array}{l}\text { PET/CT performed before treatment } \\
\text { PET/CT performed after treatment }\end{array}$ & $\begin{array}{l}55 \\
31\end{array}$ & $\begin{array}{l}63.6 \\
36.4\end{array}$ \\
\hline
\end{tabular}

for Radiology and Surgical Technologies in 2014. It has been extensively used in clinical practice since September, 2015. The device was designed using the membrane valves connected with capillaries, and adapted for generated RP. Its closest analogues are synthesis modules of Tracerlab series by General Electric, USA.

The basic advantage of our module over other analogues is the presence of a built-in syringe pump and a flow-type radioactivity sensor, which enable to separate more effectively an elute fraction with maximum specific radioactivity (process yield with the correction for cleavage is under $60 \%$ ). Moreover, RP synthesis time does not exceed $15 \mathrm{~min}$, the resulting RP is sterile and apyrogenic, radiochemical purity is not less than $95 \%$, radionuclide purity is not less than $99.9 \%$.

The technology of ${ }^{68} \mathrm{Ga}$-DOTA-TATE preparation is as follows: first, using a built-in syringe pump, ${ }^{68} \mathrm{Ge} /{ }^{68} \mathrm{Ga}$-generator is eluted using a built-in flow-type radioactivity sensor. A fraction containing maximum radionuclide (over 95\%) in an automatic mode is separated from elute followed by the reaction of labeling DOTA-TATE by gallium- 68 in a reaction vessel at $100^{\circ} \mathrm{C}$. The labeled compound was cleaned by solid-phase extraction on SepPak $\mathrm{C} 18$ cartridge. The end product is exposed to sterilization by filtration through a membrane filter, the pore diameter being $0.22 \mu \mathrm{m}$.

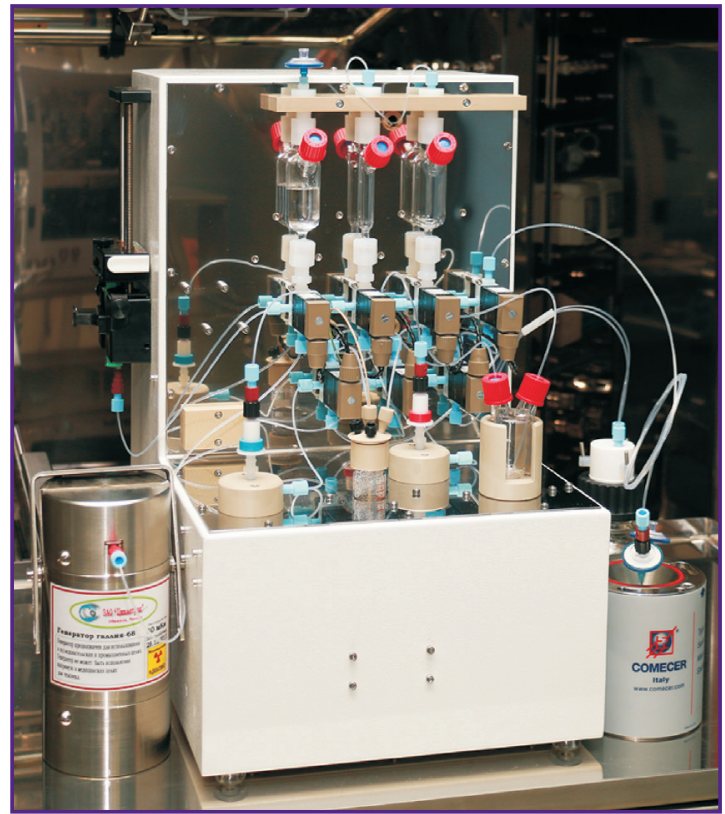

Figure 1. ${ }^{68} \mathrm{Ge} /{ }^{68} \mathrm{Ga}$-generator with a Russian synthesis module ${ }^{68} \mathrm{Ga}-\mathrm{DOTA}-\mathrm{TATE}$ - Gerat13- ${ }^{68} \mathrm{Ga}$
The patients were scanned 50-60 min after RP injection according to a whole body protocol on PET/CT device Discovery 690 (General Electric, USA). A research protocol consisted in obtaining a topogram limiting the scanning area by the orbitomeatal line and the upper third of the thigh, a low-dose CT (tube voltage $120 \mathrm{kV}$, amperage was auto selected in Smart mode, in the range 50-150 mA, tube speed $0.5 \mathrm{~s}$ ) and positron emission tomography.

In addition, to contrast intestinal loops all patients after RP administration were given $75 \%$ urografin, $20 \mathrm{ml}$, diluted in $0.5 \mathrm{~L}$ of bottled water. Moreover, in low-dose $\mathrm{CT}$, for image enhancement, all patients were given a radiopaque: Ultravist $370,50-150 \mathrm{ml}$, in accordance with a patient's body weight. CT was performed $20 \mathrm{~s}$ after radiopaque bolus dosing.

Whole body PET/CT depending on patient's height took 20-25 min. Iterated image reconstruction was an automatic mode using OSEM (Ordered Subsets Expectation Maximization) algorithm.

The processing of results included visual analysis of computed tomograms and positron emission tomograms 
separately, as well as that of the combined images. In addition, there were taken into consideration physiological RP accumulation in the pituitary gland, salivary glands, thyroid, pancreas, liver, adrenals, along the large intestine, renal calices-pelvis system and the urinary bladder. The main sign of sst-positive tumor tissue in a patient was pathological focal hyperfixation of ${ }^{68} \mathrm{Ga}-$ DOTA-TATE even if there were no evident structural organ changes according to CT. In cases when a tumor was seen on CT but at the same time it did not accumulate RP, its nature was still considered as malignant, and a tumor as sst-negative. ${ }^{68} \mathrm{Ga}$-DOTA-TATE accumulation level was estimated by calculating semi-quantitative criterion, a SUV (Standardized Uptake Value), but we did not calculate threshold SUV in the areas of its accumulation.

Results. For data systematization, and considering the period PET/CT with ${ }^{68} \mathrm{Ga}$-DOTA-TATE was performed (before and after treatment), all the patients under examination were divided into two groups. Group 1 involved 55 patients who underwent scanning before therapy, while group 2 (31 patients) underwent scanning after therapy.

$90.9 \%$ (50 of 55) patients in group 1 had focal RP hyperfixation in a primary mass in PET/CT. And in 10\% (5 of 50 ) patients a primary tumor site was unknown during
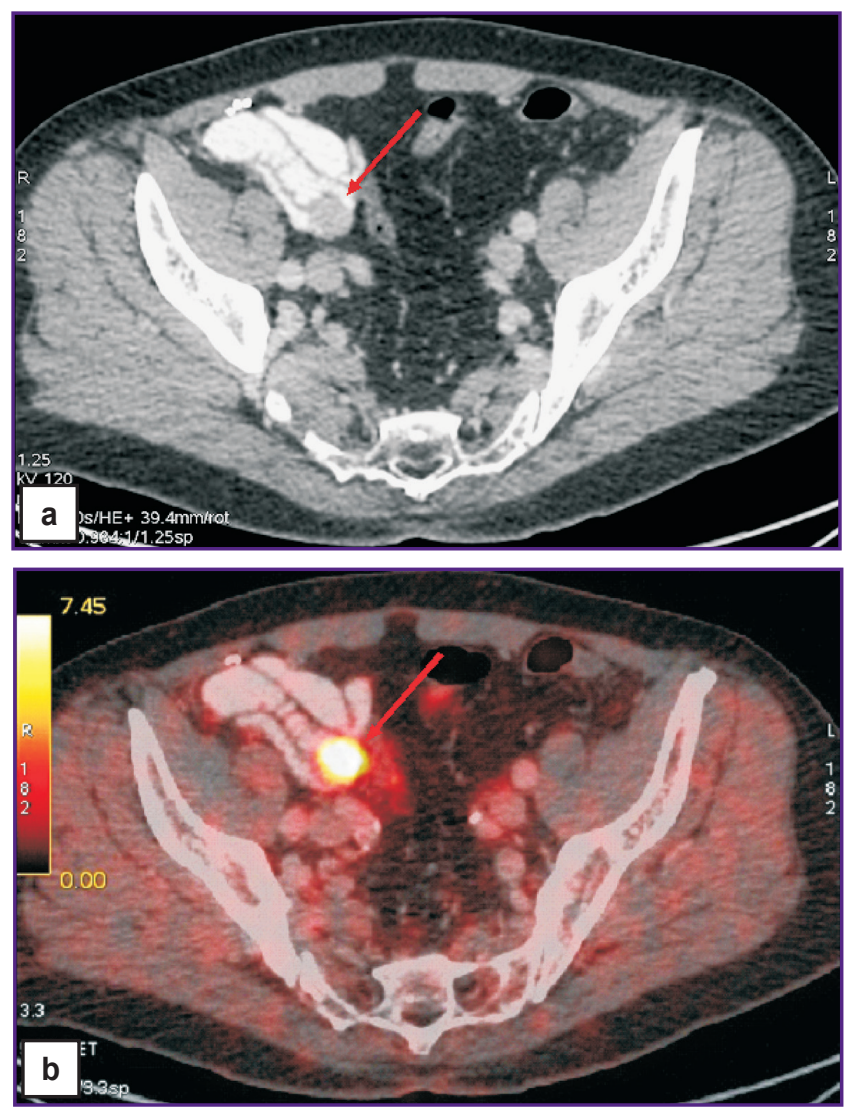

Figure 2. Findings of $C T$ (a) and combined PET/CT with ${ }^{68} \mathrm{Ga}$-DOTA-TATE (b) in a patient with neuroendocrine tumor of a terminal portion of ileum. A primary tumor focus (arrows) in the ileum was first imaged by PET/CT

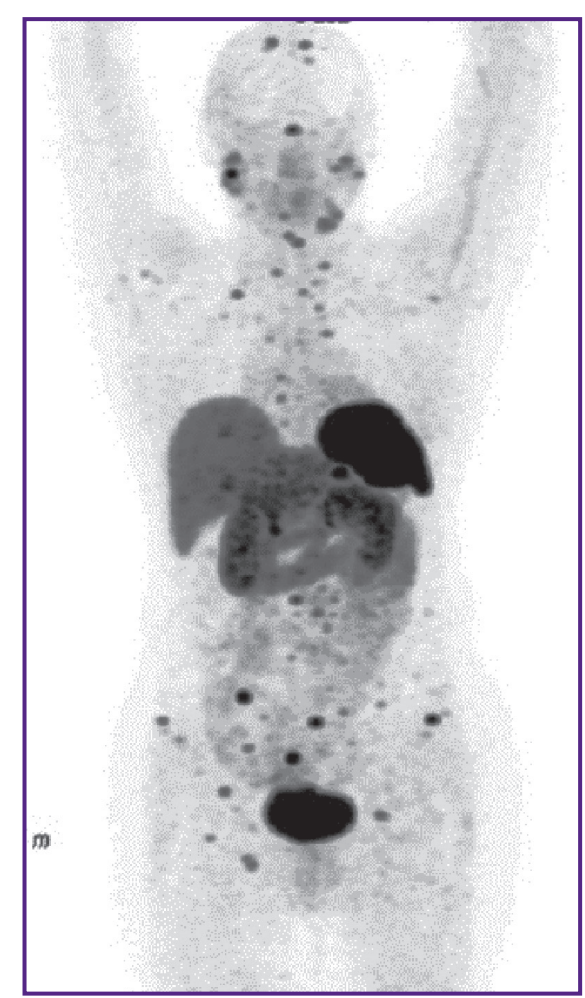

Figure 3. PET/CT with ${ }^{68} \mathrm{Ga}-\mathrm{DOTA}$-TATE findings of a female patient after surgical treatment of neuroendocrine tumor of the left proximal bronchus: numerous metastases of skeletal bones, both salivary glands, lymph nodes of the right submandibular area, the head and the tail of the pancreas, soft tissues of the right scapular region, and the anterior abdominal wall

PET/CT with ${ }^{68} \mathrm{Ga}$-DOTA-TATE. According to the findings, in 3 of 5 patients sst-positive tumor tissue was found in the pancreas, and in 2 patients - in the wall of ileum (Figure 2). First tumors detected were of small size (10$22 \mathrm{~mm}$ ), SUV levels recorded in their view varied from 6.1 to $11.7 \mathrm{~g} / \mathrm{ml}$.

In $5.5 \%$ (3 of 55 ) patients a primary tumor in the pancreas was visualized by CT only, and due to this fact it was considered as sst-negative. Subsequently, according to morphological findings of the postoperative material, these patients were diagnosed low-grade differentiated insulinoma.

In 3.6\% (2 of 55) patients a primary tumor was not

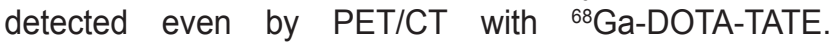
Moreover, the patients had a symptomatic complex typical for carcinoid syndrome.

In addition, regional and/or distant metastases were found by PET/CT in $85.5 \%$ (47 of 55) patients in group 1. However, the disease stage initially identified by other radiation imaging techniques was confirmed by $\mathrm{PET} / \mathrm{CT}$ only in 36 of these 47 cases (76.6\%). The rest $23.4 \%$ (11 of 47 ) patients were found to have additional secondary changes. In 6 cases metastatic foci were additionally detected in intraperitoneal and/or extraperitoneal lymph 
nodes, in 5 patients - in lymph nodes, liver parenchyma, skeletal bones, as well as other organs and tissues, and due to this fact a tumor was considered disseminated.

In 13 of 31 patients in group 2, as well as in group 1, no complete coincidence of PET/CT and clinical radiation findings was found. In 6 patients the pathological RP accumulation in a primary tumor and/or metastatic foci, contrary to good clinical radiation data suggested no positive effect of the therapy provided (Figure 3). In 3 patients secondary changes were detected after treatment in the liver only by PET/CT with ${ }^{68} \mathrm{Ga}$-DOTA-TATE. In 3 NET patients a significant increase of metastases in the liver was found only by CT. PET views of all the foci showed RP accumulation level to be within normal limits. However, despite false-negative PET results, the condition of these patients was considered as the disease progression relying on CT findings only. And finally, in one female patient after surgical treatment of NET of the left proximal bronchus against the clinical manifestations of carcinoid syndrome only PET revealed the recurrent disease in the stump of the left main bronchus. In this patient in the recurrence area CT detected just marked structural, primarily fibrous, changes.

Discussion. The analysis results of Russian and foreign suggest that interest in NET in recent years has grown significantly $[1,4,6,8,15]$. Increased attention of clinicians, morphologists, surgeons and other specialists to the problem is related primarily to an increased detection rate and the peculiarities of biological behavior of NET. It should be emphasized that a considerable part of debaters on the problem consider optimal planning of diagnostic measures before primary treatment to be one of the most important components of successful treatment of NET patients. Over the last years, combined PET/CT with novel receptor specific RP ranks a prominent position among other current radio diagnostic techniques.

Generally, we consider our first experience of production and clinical application of ${ }^{68} \mathrm{Ga}$-DOTA-TATE to be successful. Using RP synthesis module developed by our radiochemists we obtained 86 diagnostic doses ${ }^{68} \mathrm{Ga}$-DOTA-TATE ready for administration. RP application in most patients with NET enabled to image a primary tumor and its metastases. Moreover, ${ }^{68} \mathrm{Ga}$-DOTA-TATE appeared to be very effective in the assessment of the therapy provided. In addition, in the future we are to gain insight into ${ }^{68} \mathrm{Ga}$-DOTA-TATE applicability to diagnose NET localizing in other organs, which have not been considered in our study, as well as its pharmacodynamics in non-tumor diseases, first of all of inflammatory nature.

Conclusion. Positron emission tomography with ${ }^{68} \mathrm{Ga}-$ DOTA-TATE showed high efficacy in detecting a primary tumor, estimating the therapy results and dissemination degree of neuroendocrine tumors.

Study Funding. The study was not funded by any sources.

Conflicts of Interest. The authors have no conflicts of interest related to the present study.

\section{References}

1. Yao J.C., Hassan M., Phan A., Dagohoy C., Leary C., Mares J.E., Abdalla E.K., Fleming J.B., Vauthey J.N., Rashid A., Evans D.B. One hundred years after "carcinoid": epidemiology of and prognostic factors for neuroendocrine tumors in 35,825 cases in the United States. J Clin Oncol 2008; 26(18): 3063-3072, https://doi.org/10.1200/ jco.2007.15.4377.

2. Hauso O., Gustafsson B.I., Kidd M., Waldum H.L., Drozdov I., Chan A.K., Modlin I.M. Neuroendocrine tumor epidemiology: contrasting Norway and North America. Cancer 2008; 113(10): 2655-2664, https://doi.org/10.1002/ cncr. 23883 .

3. Stridsberg M., Oberg K., Li Q., Engstrom U., Lundqvist $\mathrm{G}$. Measurements of chromogranin A, chromogranin B (secretogranin I), chromogranin C (secretogranin II) and pancreastatin in plasma and urine from patients with carcinoid tumours and endocrine pancreatic tumours. J Endocrinol 1995; 144(1): 49-59, https://doi.org/10.1677/joe.0.1440049.

4. Barakat M.T., Meeran K., Bloom S.R. Neuroendocrine tumours. Endocr Relat Cancer 2004; 11(1): 1-18, https://doi. org/10.1677/erc.0.0110001.

5. Kasprzak A., Zabel M., Biczysko W. Selected markers (chromogranin A, neuron-specific enolase, synaptophysin, protein gene product 9.5) in diagnosis and prognosis of neuroendocrine pulmonary tumours. Pol J Pathol 2007; 58(1): 23-33.

6. Kulke M.H. Neuroendocrine tumours: clinical presentation and management of localized disease. Cancer Treat Rev 2003; 29(5): 363-370, https://doi.org/10.1016/ s0305-7372(03)00072-0.

7. Rindi G., Klöppel G. Endocrine tumors of the gut and pancreas tumor biology and classification. Neuroendocrinology 2004; 80(Suppl 1): 12-15, https://doi. org/10.1159/000080733.

8. Tlostanova M.S., Petrunkin A.M. The effectiveness of pet with ${ }^{18} \mathrm{~F}$-fluorodesoxyglucose in the diagnosis of lung neuroendocrine tumors. Voprosy onkologii 2013; 59(4): 505-508.

9. Abgral R., Leboulleux S., Déandreis D., Aupérin A., Lumbroso J., Dromain C., Duvillard P., Elias D., de Baere T., Guigay J., Ducreux M., Schlumberger M., Baudin E. Performance of 18fluorodeoxyglucose-positron emission tomography and somatostatin receptor scintigraphy for high Ki67 ( $\geq 10 \%)$ well-differentiated endocrine carcinoma staging. J Clin Endocrinol Metab 2011; 96(3): 665-671, https://doi. org/10.1210/jc.2010-2022.

10. Öberg K., Hellman P., Kwekkeboom D., Jelic S.; ESMO Guidelines Working Group. Neuroendocrine bronchial and thymic tumours: ESMO Clinical Practice Guidelines for diagnosis, treatment and follow-up. Ann Oncol 2010; 21(Suppl 5): v220-v222, https://doi.org/10.1093/annonc/mdq191.

11. Severi S., Nanni O., Bodei L., Sansovini M., lanniello A., Nicoletti S., Scarpi E., Matteucci F., Gilardi L., Paganelli G. Role of ${ }^{18} \mathrm{FDG}$ PET/CT in patients treated with ${ }^{177} \mathrm{Lu}$-DOTATATE for advanced differentiated neuroendocrine tumours. Eur J Nucl Med Mol Imaging 2013; 40(6): 881-888, https://doi. org/10.1007/s00259-013-2369-z.

12. Kodina G.E., Kozlova M.D., Krasnov N.N., Malinin A.B., Sevast'yanov Yu.G., Sevast'yanova A.S., Razbash A.A., Sharygin L.M. Radionuklidnyy generator $68 \mathrm{Ge} / 68 \mathrm{Ga}$ dlya polucheniya fiziologicheski priemlemogo rastvora 
[Radionuclide generator $68 \mathrm{Ge} / 68 \mathrm{Ga}$ for preparing a physically acceptable solution]. Patent RF No.2126271. 1998.

13. Larenkov A.A., Kodina G.E., Bruskin A.B. Gallium radionuclides in nuclear medicine: radiopharmaceuticals based on $68 \mathrm{Ga}$. Meditsinskaya radiologiya $i$ radiatsionnaya bezopasnost' 2011; 56(5): 56-73.

14. Wild D., Bomanji J.B., Benkert P., Maecke H., Ell P.J., Reubi J.C., Caplin M.E. Comparison of ${ }^{68} \mathrm{Ga}-$ DOTANOC and ${ }^{68} \mathrm{Ga}$-DOTATATE PET/CT within patients with gastroenteropancreatic neuroendocrine tumors. J Nucl Med 2013; 54(3): 364-372, https://doi.org/10.2967/ jnumed.112.111724.
15. Poeppel T.D., Binse I., Petersenn S., Lahner H., Schott M., Antoch G., Brandau W., Bockisch A., Boy C. Differential uptake of ${ }^{68} \mathrm{Ga}$-DOTATOC and ${ }^{68} \mathrm{Ga}$-DOTATATE in PET/CT of gastroenteropancreatic neuroendocrine tumors. Recent Results Cancer Res 2013; 194: 353-371, https://doi. org/10.1007/978-3-642-27994-2_18.

16. Poeppel T.D., Binse I., Petersenn S., Lahner H., Schott M., Antoch G., Brandau W., Bockisch A., Boy C. ${ }^{68} \mathrm{Ga}-\mathrm{DOTATOC}$ versus ${ }^{68} \mathrm{Ga}$-DOTATATE PET/CT in functional imaging of neuroendocrine tumors. J Nucl Med 2011; 52(12): 1864-1870, https://doi.org/10.2967/jnumed. 111.091165. 